\title{
O uso de plantas medicinais na atenção primária à saúde: revisão integrativa
}

\author{
Medicinal plant use in primary health care: an integrative review
}

Karina Pavão Patrício (https://orcid.org/0000-0003-2112-5956) ${ }^{1}$

Arthur Cesar dos Santos Minato (https://orcid.org/0000-0003-2484-8631) ${ }^{2}$

Ana Flavia Brolio (https://orcid.org/0000-0002-3666-9473) ${ }^{2}$

Marina Amorim Lopes (https://orcid.org/0000-0001-5241-2486) ${ }^{2}$

Gabriela Ribeiro de Barros (https://orcid.org/0000-0002-2984-9820) ${ }^{2}$

Vanessa Moraes (https://orcid.org/0000-0003-2645-0563) ${ }^{2}$

Guilherme Correa Barbosa (https://orcid.org/0000-0002-7433-8237) ${ }^{3}$
${ }^{1}$ Departamento de Saúde Pública, Faculdade de Medicina de Botucatu, Universidade Estadual Paulista "Júlio de Mesquita Filho". Av. Prof. Mário Rubens Guimarães

Montenegro $s / \mathrm{n}$, Campus de Botucatu. 18618-687 Botucatu SP Brasil. karina. pavao@unesp.br

${ }^{2}$ Faculdade de Medicina de Botucatu, Universidade Estadual Paulista "Júlio de Mesquita Filho". Botucatu SP Brasil.

${ }^{3}$ Departamento de Enfermagem. Faculdade de Medicina de Botucatu, Universidade Estadual

Paulista "Júlio de Mesquita Filho". Botucatu SP Brasil.

\begin{abstract}
Medicinal plant (MP) use supports comprehensiveness of care in Primary Health Care (PHC), enabling appreciation of popular knowledge and self-care. This integrative literature review aims to analyze researches that approach the insertion of using MP in PHC. PICO strategy was used as a guideline in search of evidence, reuniting 18 articles published between January 2015 and August 2020, in the Virtual Health Library and PubMed databases. The variables of analysis were knowledge of PHC healthcare professionals about MP and associated policies, MP use by its users, highlighting their profile, the reasons that lead to the use and lack of security in MP use. The results show insufficient knowledge of healthcare professionals about Integrative and Complementary Practices policies and the medicinal uses of plants. The main users are women, elderly, with low income and education, either in Brazil or other countries. Regarding safety in MP use, frequently there is no correct identification of species, its origin, its preparation and the appropriate dose for each case. Finally, failure to approach these contents during training of healthcare professionals generates less knowledge, less research and more prejudice due to lack of information, impairing incentive and dissemination to the community. Key words Medicinal plants, Medicinal herbs, Primary health care
\end{abstract}

Resumo O uso de plantas medicinais (PM) favorece a integralidade do cuidado na atenção primária à saúde (APS), valorizando o saber popular e o autocuidado. Esta revisão integrativa de literatura objetiva analisar estudos sobre a inserção do uso de PM na APS. A estratégia PICO norteou a busca de evidências, reunindo 18 artigos publicados de janeiro de 2015 a agosto de 2020 nos bancos de dados Biblioteca Virtual de Saúde e PubMed. As variáveis de análise foram o conhecimento dos profissionais da saúde da APS sobre PM e politicas associadas, o uso de PM pelos usuários, destacando seu perfil, fatores que favorecem o uso e a falta de segurança no uso de PM. A literatura aponta insuficiência de conhecimento dos profissionais de saúde sobre as políticas de práticas integrativas e complementares e o uso de plantas para fins medicinais. Os principais usuários são mulheres, idosas, com baixa renda e escolaridade, tanto no Brasil quanto em outros países. Sobre a segurança no uso de PM, frequentemente não há correta identificação de espécie, origem, preparo e dose adequada para cada caso. Por fim, a não abordagem desses conteúdos durante a formação de profissionais da saúde gera menos conhecimento, menos pesquisas e mais preconceito por falta de informação, prejudicando o incentivo e divulgação à comunidade.

Palavras-chave Plantas medicinais, Ervas medicinais, Atenção primária à saúde 


\section{Introdução}

É histórico o uso de diferentes plantas a favor de objetivos humanos, desde a aplicação como venenos até o emprego em atividades curativas ${ }^{1}$, sendo, no último caso, denominada planta medicinal (PM). Esse uso foi inspirado na observação empírica de práticas de outros animais, uma vez que várias espécies apresentavam hábitos de consumir plantas mediante situação de injúrias².

Por meio de tal análise, os seres humanos começaram a sistematizar o uso de PM, conciliando aspectos filosóficos e culturais característicos, desenvolvendo a base de vertentes da dedicina ${ }^{3}$. Assim, considera-se PM aquela capaz de aliviar ou curar enfermidades e que tem tradição de uso como remédio pelas pessoas da comunidade ${ }^{4}$.

Com o passar dos séculos, o cuidado com a saúde sofreu transformações. Hoje se valoriza o conhecimento científico acadêmico e o modelo biomédico centrado na doença, em detrimento aos outros tipos de geração de conhecimentos. Assim, surge a medicalização da vida, um fenômeno de apropriação pela medicina, que interfere na construção e interpretação de conceitos, costumes e comportamentos sociais ${ }^{6}$. Há, nesse contexto, desvalorização do autocuidado e da conexão com a natureza, com valorização cada vez maior da medicina convencional moderna como único recurso eficaz e seguro ${ }^{5,6}$.

Por outro lado, surgiram nas últimas décadas movimentos sociais e políticas públicas que buscam resgatar os saberes tradicionais e valorizar a promoção e integralidade no cuidado à saúde, estimulando a interação humanidade-natureza ${ }^{7}$.

Em 1978, com a declaração de Alma-Ata, a Organização Mundial da Saúde (OMS) passou a reconhecer o uso de PM e fitoterápicos como estratégias efetivas em saúde, validando suas propriedades curativas, profiláticas e paliativas ${ }^{8}$. No Brasil, a temática foi abordada na $8^{a}$ Conferência Nacional ${ }^{9}$, em 1986, definindo-se que práticas alternativas de assistência à saúde deveriam ser parte do Sistema Único de Saúde (SUS), ainda em construção, permitindo ao usuário escolher a terapêutica desejada.

Passados 20 anos, com a regulamentação da Política Nacional de Práticas Integrativas e Complementares (PNPIC) $)^{10}$ e da Política Nacional de Plantas Medicinais e Fitoterápicos ${ }^{11}$, determinou-se a adoção do uso de PM e da fitoterapia no SUS, com enfoque na atenção primária à saúde (APS), como mais uma forma de tratamento para processos de adoecimento da população, valorizando também o saber popular ${ }^{12}$.
Por meio de políticas e movimentos que fortalecem as práticas integrativas e complementares, objetiva-se o resgate de saberes populares, favorecendo formas de cuidado holístico que promovam sustentabilidade, valorização do autocuidado e participação ativa do paciente em todo o processo. Com uso de PM, é possível alcançar esses objetivos, fomentando ainda a educação ambiental, de modo a promover a saúde e o resgate do autocuidado ${ }^{13}$.

Dessa forma, o objetivo deste estudo foi analisar pesquisas que abordaram formas de uso de PM na APS, caracterizando o perfil desses usuários, fatores que promovem o uso, qual o conhecimento dos profissionais da saúde sobre o tema e as políticas associadas, bem como a questão da falta de segurança e assistência adequadas quanto ao uso de PM na APS.

\section{Método}

Trata-se de uma revisão integrativa da literatura, instrumento de obtenção, identificação, análise e síntese das publicações acerca de um tema específico. Foram seguidas as seguintes etapas: identificação do problema (definindo o tema da revisão em forma de questão, denominada pergunta PICO ou norteadora); seleção da amostra, definição das características da pesquisa (categorização dos estudos e da coleta de dados); análise dos estudos incluídos na revisão, identificando similaridades e conflitos; discussão e interpretação dos resultados; apresentação da revisão/síntese do conhecimento ${ }^{14,15}$.

Para a elaboração desta revisão integrativa, partiu-se da pergunta: quais as evidências científicas relacionadas com as formas de uso de plantas medicinais na atenção primária à saúde?

Para a busca nos bancos de dado selecionados (Biblioteca Virtual em Saúde - BVS, e US National Library of Medicine - PubMed), utilizou-se as seguintes palavras-chave: plantas medicinais; ervas medicinais e relação nacional de plantas medicinais; atenção primária à saúde; atendimento básico; atendimento primário; atendimento primário de saúde; atenção básica; atenção básica de saúde; atenção básica à saúde; atenção primária; atenção primária de saúde; atenção primária em saúde; cuidados primários; cuidados primários de saúde; cuidados primários à saúde; cuidados de saúde primários; primeiro nível de assistência; primeiro nível de atendimento; primeiro nível de atenção; primeiro nível de atenção à saúde; primeiro nível de cuidado; primeiro nível de cuidados. 
A busca de artigos foi realizada por quatro pesquisadores, utilizando-se dos mesmos critérios, sendo dois responsáveis pela pesquisa na base de dados BVS e dois na base PubMed. Realizou-se duplicata, às cegas, validando a metodologia. Dois pesquisadores foram responsáveis pela seleção dos artigos. Em caso de divergência, optou-se por manter os artigos na seleção até a etapa de leitura integral, verificando se esses contemplavam o tema desta revisão.

A seleção da amostra inicial adotou como critérios de inclusão artigos que trouxessem em seus títulos ou resumos elementos sobre utilização de PM na APS, encontrados nas literaturas nacional e internacional, publicados no período de janeiro de 2015 a agosto de 2020, nos idiomas português, espanhol e inglês. Foram excluídas teses de dissertação e revisões e aceitou-se artigos com diversos delineamentos metodológicos, pelo fato de a questão norteadora exigir abrangência acerca da temática investigada.

Com isso, a amostragem constituiu-se de 1.038 artigos, sendo 533 encontrados na base de dados BVS e 505 artigos na PubMed, com a aplicação dos filtros de idioma e data. Foram excluídos 981 pelas seguintes causas: títulos ou resumos não se adequarem à pergunta tema ou por se tratar de revisões ou teses de dissertação, restando 57 artigos (Figura 1).
Nessa etapa, todos os 57 artigos estavam disponíveis para leitura: 14 de acesso público e quatro privados, acessados por meio da universidade apoiadora desta pesquisa.

Foram descartados 11 artigos duplicados, às cegas, sobrando 46. Todos esses foram lidos na íntegra e 28 foram excluídos por abordarem de forma pouco exploratória o uso de PM na APS, não respondendo à pergunta. Por meio da análise de dois pesquisadores, 18 artigos foram estabelecidos como corpus de análise, sendo dez da base de dados BVS e oito da PubMed (Figura 1).

Para o estudo dos artigos, foi utilizada a técnica de análise temática ${ }^{16}$, que consiste nos seguintes passos: leitura flutuante do conteúdo, codificação, categorização e articulação com os seguintes referenciais teóricos - Política Nacional de Práticas Integrativas e Complementares $(\mathrm{PNPIC})^{10}$ e Política Nacional de Plantas Medicinais e Fitoterápicos ${ }^{11}$.

Foram criadas, então, as seguintes categorias: "Conhecimento dos profissionais da saúde da APS sobre PM e políticas associadas", "Usuários de PM na APS: perfil e fatores que levam ao uso" e "A falta de segurança no uso de PM". Fez-se a leitura criteriosa de cada artigo, observando essas categorias, podendo o mesmo artigo estar presente em mais de uma seção.
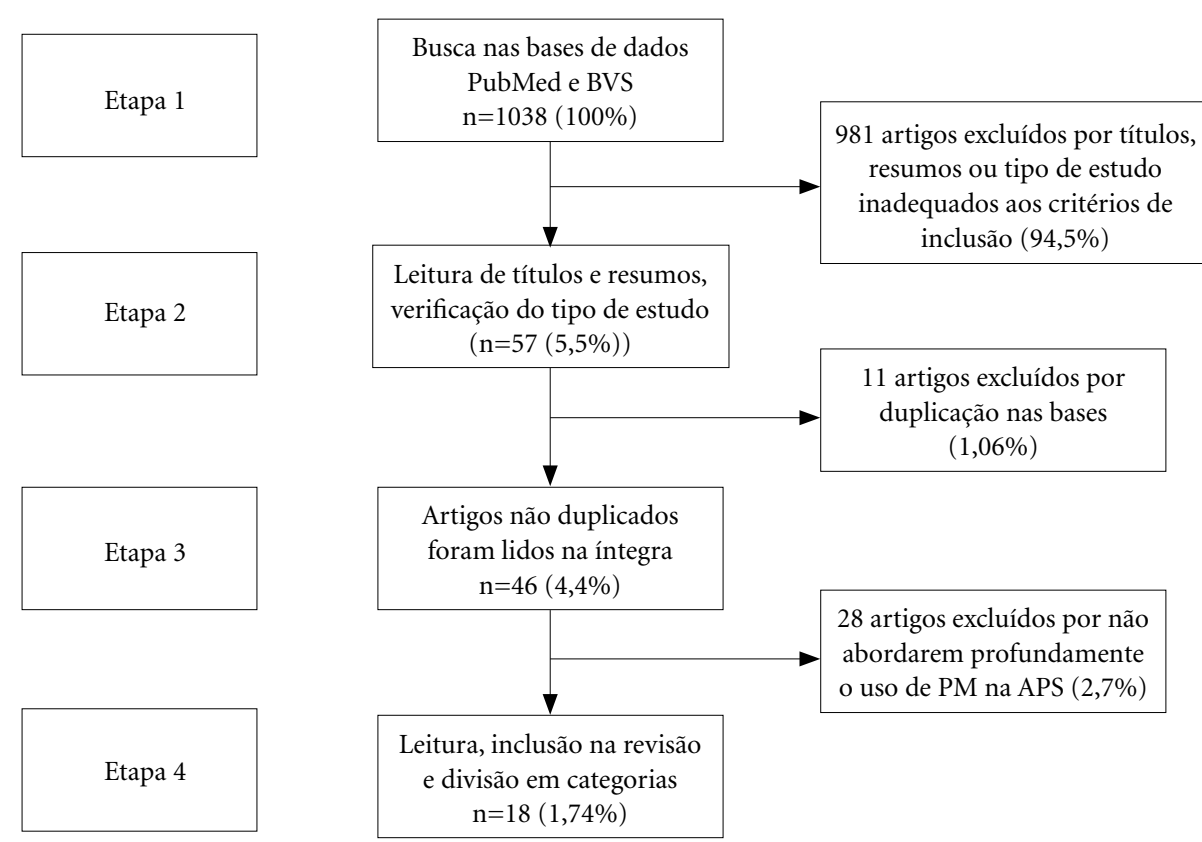

28 artigos excluídos por não abordarem profundamente o uso de PM na APS (2,7\%)

Figura 1. Fluxograma referente aos resultados de busca. 


\section{Resultados e discussão}

Dos 18 artigos selecionados para análise, sete foram elaborados em outros países, a saber: Peru, Paraguai, Espanha, México, Jamaica, Paquistão e Sérvia. Oito foram publicados exclusivamente em português, quatro em inglês, três em espanhol e três tanto em português como em inglês. Destacam-se a Revista Ciência e Saúde Coletiva (n =3) e o Journal of Ethnopharmacology $(\mathrm{n}=4)$ com as maiores quantidades de artigos publicados na temática desta revisão.

Os estudos foram descritos no Quadro 1, mostrando autores, ano de publicação, local da pesquisa, revista publicada, tipo de estudo e as principais evidências encontradas.

A partir dos resultados, evidenciou-se a importância de se discutir diferentes variáveis que influenciam no uso de PM na APS, mostrando a complexidade das questões que permeiam o cuidado em saúde. Dessa forma, destacamos temáticas que serviram como base de análise, possibilitando a relação entre os dados e o aprofundamento na literatura para compreensão do nível de conhecimento dos profissionais frente à PM, à caracterização dos usuários e à utilização das $\mathrm{PM}$ com maior segurança, a fim de se responder à pergunta PICO.

Quadro 1. Distribuição dos artigos selecionados segundo autores, ano de publicação, local da pesquisa, revista publicada, tipo de estudo e principais evidências encontradas.

\begin{tabular}{|c|c|c|}
\hline Autor/ano & Revista/local/tipo de estudo & Principais evidências do estudo \\
\hline $\begin{array}{l}\text { Dantas et } \\
\text { al., } 2019\end{array}$ & $\begin{array}{l}\text { Revista de Saúde Coletiva (PE - } \\
\text { Brasil); estudo qualitativo }\end{array}$ & $\begin{array}{l}\text { As práticas de saúde se relacionaram ao cuidado popular } \\
\text { por meio do uso de PM e ações de prevenção; fatores } \\
\text { socioeconômicos, culturais e educacionais da comunidade } \\
\text { impactaram negativamente a condição de saúde; observou-se } \\
\text { discrepância entre o fazer saúde técnico e o popular }\end{array}$ \\
\hline $\begin{array}{l}\text { Soares et } \\
\text { al., } 2019\end{array}$ & $\begin{array}{l}\text { Revista de Enfermagem do } \\
\text { Centro-Oeste Mineiro (MG - } \\
\text { Brasil); estudo descritivo com } \\
\text { abordagem qualitativa }\end{array}$ & $\begin{array}{l}\text { Desconhecimento dos enfermeiros em relação à PNPIC devido } \\
\text { às lacunas no processo de formação, capacitação e educação } \\
\text { permanente dos profissionais. Apesar disso, os profissionais } \\
\text { apontaram como importante fonte de cuidado o uso de PM e } \\
\text { fitoterápicos, mas não sabem diferenciar os mesmos }\end{array}$ \\
\hline $\begin{array}{l}\text { Valenzuela- } \\
\text { Ore et al., } \\
2018\end{array}$ & $\begin{array}{l}\text { Revista Peruana de Medicina } \\
\text { Experimental y Salud Pública, } \\
\text { (Huancavelica - Peru); estudo } \\
\text { transversal com abordagem } \\
\text { quantitativa }\end{array}$ & $\begin{array}{l}\text { Apesar de as comunidades analisadas fazerem grande uso dos } \\
\text { serviços formais do sistema de saúde, as práticas culturais e } \\
\text { populares ainda são bastante prevalentes, destacando-se o uso } \\
\text { de PM }\end{array}$ \\
\hline $\begin{array}{l}\text { Caccia-Bava } \\
\text { et al., } 2017\end{array}$ & $\begin{array}{l}\text { Revista Ciência e Saúde } \\
\text { Coletiva (SP - Brasil); estudo } \\
\text { transversal e multicêntrico, com } \\
\text { abordagem qualiquantitativa }\end{array}$ & $\begin{array}{l}\text { A disponibilidade do fitoterápico é maior em municípios } \\
\text { mais populosos e com melhores índices socioeconômicos. } \\
\text { Constatou-se também que o fitoterápico industrializado é } \\
\text { mais utilizado e aceito do que a droga vegetal in natura ou o } \\
\text { fitoterápico manipulado }\end{array}$ \\
\hline $\begin{array}{l}\text { Szerwieski } \\
\text { et al., } 2017\end{array}$ & $\begin{array}{l}\text { Revista Eletrônica de } \\
\text { Enfermagem (PR - Brasil); } \\
\text { estudo transversal, } \\
\text { correlacional, analítico e } \\
\text { descritivo, com abordagem } \\
\text { quantitativa }\end{array}$ & $\begin{array}{l}\text { O uso de PM por idosos é bem difundido na população } \\
\text { analisada, mas é necessário orientá-los corretamente quanto } \\
\text { à toxicidade e aos benefícios de algumas plantas, haja vista o } \\
\text { desconhecimento generalizado. O enfermeiro atua como um } \\
\text { importante promotor de saúde nesse contexto }\end{array}$ \\
\hline $\begin{array}{l}\text { Souza et al., } \\
2016\end{array}$ & $\begin{array}{l}\text { Revista Brasileira de Plantas } \\
\text { Medicinais (RS - Brasil); estudo } \\
\text { qualitativo tipo descritivo }\end{array}$ & $\begin{array}{l}\text { Observou-se desconhecimento da Política, embora o uso de PM } \\
\text { no meio de trabalho seja bem aceito. A Política é mais efetiva } \\
\text { quando o enfermeiro organiza grupos, põe em prática o diálogo } \\
\text { interdisciplinar e contempla os aspectos culturais do usuário }\end{array}$ \\
\hline $\begin{array}{l}\text { Palma et al., } \\
2015\end{array}$ & $\begin{array}{l}\text { Revista de Pesquisa Cuidado } \\
\text { é Fundamental Online, (RS - } \\
\text { Brasil); estudo qualitativo }\end{array}$ & $\begin{array}{l}\text { Os profissionais da saúde utilizam PM como ferramenta de } \\
\text { autocuidado, e acreditam ser possível incorporar o uso de PM } \\
\text { na APS em situações de autocuidado e cuidados paliativos. } \\
\text { Fora isso, há preferência por recursos alopáticos convencionais, } \\
\text { explicitando um reflexo da formação profissional embasada no } \\
\text { modelo biomédico }\end{array}$ \\
\hline
\end{tabular}


Quadro 1. Distribuição dos artigos selecionados segundo autores, ano de publicação, local da pesquisa, revista publicada, tipo de estudo e principais evidências encontradas.

\begin{tabular}{|c|c|c|}
\hline Autor/ano & Revista/local/tipo de estudo & Principais evidências do estudo \\
\hline $\begin{array}{l}\text { Colet et al., } \\
2015\end{array}$ & $\begin{array}{l}\text { Revista Brasileira de Medicina } \\
\text { de Família e Comunidade (RS } \\
\text { - Brasil); estudo transversal de } \\
\text { abordagem quantitativa }\end{array}$ & $\begin{array}{l}\text { Explicitou-se a necessidade de capacitação dos profissionais da } \\
\text { saúde para uma correta orientação do usuário no uso de PM, } \\
\text { bem como para o fomento à pesquisa com PM para embasar a } \\
\text { implementação de políticas públicas de fitoterapia }\end{array}$ \\
\hline $\begin{array}{l}\text { Soria et al., } \\
2015\end{array}$ & $\begin{array}{l}\text { Memorias del Instituto de } \\
\text { Investigaciones em Ciencias de } \\
\text { la Salud (Assunção - Paraguai); } \\
\text { estudo observacional, descritivo, } \\
\text { transversal e prospectivo de } \\
\text { aspecto qualiquantitativo }\end{array}$ & $\begin{array}{l}\text { Evidenciou-se a necessidade de se educar os profissionais da } \\
\text { saúde e a população para uma correta identificação das PM } \\
\text { utilizadas pela população paraguaia, a fim de se conhecer o } \\
\text { potencial de toxicidade de cada planta }\end{array}$ \\
\hline $\begin{array}{l}\text { Mattos et } \\
\text { al., } 2018\end{array}$ & $\begin{array}{l}\text { Ciência e Saúde Coletiva (SC - } \\
\text { Brasil); estudo quantitativo e de } \\
\text { corte transversal }\end{array}$ & $\begin{array}{l}\text { Grande parte dos profissionais da saúde relatou conhecer } \\
\text { a PNPIC, embora a maioria desconhecesse a presença de } \\
\text { fitoterápicos na Relação Nacional de Medicamentos Essenciais. } \\
\text { Os profissionais acreditam no potencial das PM, mas não as } \\
\text { prescrevem. Contudo, concordam com a iniciativa de ofertá-las } \\
\text { no SUS após adequada capacitação }\end{array}$ \\
\hline $\begin{array}{l}\text { Zeni et al., } \\
2017\end{array}$ & $\begin{array}{l}\text { Ciência e Saúde Coletiva (SC - } \\
\text { Brasil); estudo epidemiológico } \\
\text { observacional, do tipo seccional, } \\
\text { de aspecto quantitativo }\end{array}$ & $\begin{array}{l}\text { As PM são utilizadas como alternativa terapêutica, contudo é } \\
\text { necessário que os serviços da APS garantam o acesso a esses } \\
\text { produtos, bem como a adequada qualificação dos profissionais }\end{array}$ \\
\hline $\begin{array}{l}\text { Maciá et al., } \\
2016\end{array}$ & $\begin{array}{l}\text { Revista de Fitoterapia, } \\
\text { (Barcelona - Espanha); estudo } \\
\text { transversal descritivo, de } \\
\text { abordagem quantitativa }\end{array}$ & $\begin{array}{l}\text { O uso de PM é bastante aceito e difundido na comunidade. } \\
\text { Assim, deve haver um controle adequado dessa prática nos } \\
\text { serviços de saúde pública, a fim de avaliar e evitar possíveis } \\
\text { interações medicamentosas e efeitos colaterais }\end{array}$ \\
\hline $\begin{array}{l}\text { Alonso- } \\
\text { Castro et } \\
\text { al., } 2017\end{array}$ & $\begin{array}{l}\text { Journal of Ethnopharmacology } \\
\text { (México); estudo quantitativo }\end{array}$ & $\begin{array}{l}\text { As PM são muito utilizadas por profissionais da saúde na APS } \\
\text { no México, com boa aceitação da comunidade local }\end{array}$ \\
\hline $\begin{array}{l}\text { Picking et } \\
\text { al., } 2015\end{array}$ & $\begin{array}{l}\text { Journal of Ethnopharmacology } \\
\text { (Jamaica); pesquisas } \\
\text { bibliográficas, estudos } \\
\text { experimentais, com abordagem } \\
\text { qualitativa }\end{array}$ & $\begin{array}{l}\text { O uso de PM desempenha papel fundamental na APS da } \\
\text { Jamaica no tratamento e prevenção de diversas doenças }\end{array}$ \\
\hline $\begin{array}{l}\text { Tribess et } \\
\text { al., } 2015\end{array}$ & $\begin{array}{l}\text { Journal of Ethnopharmacology } \\
\text { (Sul do Brasil); estudo } \\
\text { quantitativo }\end{array}$ & $\begin{array}{l}\text { A medicina tradicional mostra-se um importante recurso da } \\
\text { APS em comunidades rurais na região da Mata Atlântica. Nessa } \\
\text { região, há um maior conhecimento sobre o uso de PM do que } \\
\text { em outras localidades. Tal forma de conhecimento tradicional } \\
\text { representa estratégias importantes na educação ambiental, } \\
\text { preservação do meio ambiente e na validação do uso de PM } \\
\text { em políticas de saúde que beneficiem a população }\end{array}$ \\
\hline $\begin{array}{l}\text { Yaseen et } \\
\text { al., } 2015\end{array}$ & $\begin{array}{l}\text { Journal of Ethnopharmacology } \\
\text { (deserto Thar, Sindh, } \\
\text { Paquistão); estudo quantitativo. }\end{array}$ & $\begin{array}{l}\text { O uso de PM representa, na região estudada, uma fonte } \\
\text { importante de autocuidado na prática da APS dentro de } \\
\text { comunidades rurais }\end{array}$ \\
\hline $\begin{array}{l}\text { Starosta et } \\
\text { al., } 2020\end{array}$ & $\begin{array}{l}\text { Rev Eletron Comum InfI nov } \\
\text { Saúde (PR - Brasil); relato de } \\
\text { experiência com abordagem } \\
\text { qualitativa }\end{array}$ & $\begin{array}{l}\text { O documentário atua como propagador dos conhecimentos } \\
\text { tradicionais reunidos nas entrevistas, facilitando a divulgação } \\
\text { das informações para pesquisadores, profissionais e diferentes } \\
\text { comunidades, favorecendo o diálogo e a troca de saberes }\end{array}$ \\
\hline $\begin{array}{l}\text { Živković et } \\
\text { al., } 2020\end{array}$ & $\begin{array}{l}\text { Frontiers in Pharmacology, } \\
\text { (distrito de Pčinja - Sérvia); } \\
\text { estudo quantitativo }\end{array}$ & $\begin{array}{l}\text { As PM são muito utilizadas como modalidade de APS na } \\
\text { região estudada, principalmente no tratamento de doenças } \\
\text { menos complexas. Entretanto, as PM possuem um amplo } \\
\text { espectro de uso, o que deve ser mais explorado }\end{array}$ \\
\hline
\end{tabular}




\section{Conhecimento dos profissionais da saúde da APS sobre PM e políticas associadas}

Estudos apontam a insuficiência de conhecimento dos profissionais de saúde em relação às políticas de práticas integrativas e complementares e aos aspectos gerais do uso das PM. Em pesquisa realizada em 45 unidades de Estratégia Saúde da Família no município de Blumenau (SC), 65,6\% dos profissionais de saúde relataram conhecer a PNPIC, porém não souberam citar todas as práticas, demonstrando conhecimento superficial da mesma ${ }^{17}$.

Por outro lado, estudos realizados entre enfermeiros indicaram que $88,7 \%$ dos entrevistados da região Sul do estado do RS possuíam déficit de conhecimentos das políticas nacionais e públicas de valorização do uso e difusão de terapias complementares, incluindo a Política Nacional de Plantas Medicinais e Fitoterápicos ${ }^{18,19}$.

$\mathrm{O}$ não aprofundamento nos conhecimentos de tais políticas pode implicar não adesão às PIC, resultando na desvalorização dessa forma de cuidado. Assim, é necessário que os profissionais adquiram esses conhecimentos para a consolidação da política ${ }^{18}$.

Autores destacam que, durante a formação, profissionais da saúde não aprendem sobre medicina tradicional e muitas vezes desconhecem evidências científicas acerca das $\mathrm{PM}^{20,21}$. Pesquisa realizada junto a nove profissionais de uma Unidade de Saúde da Família (USF) da zona rural do município de Pelotas (RS) evidencia que, entre os profissionais participantes, nenhum teve disciplinas ou discussões durante a trajetória acadêmica que abordasse o tema de $\mathrm{PM}^{21}$.

O desconhecimento sobre PM acarreta também insegurança dos profissionais de saúde em prescrevê-las, como evidenciado em dados apresentados por Mattos e colaboradores ${ }^{17}$, no qual os entrevistados desconheciam as plantas ou os fitoterápicos contidos na Relação Nacional de Medicamentos Essenciais (RENAME).

Por outro lado, $93 \%$ dos profissionais desse mesmo estudo possuíam conhecimento sobre medicina tradicional por influências e experiências familiares, com $84,7 \%$ já tendo prescrito ou sugerido, no caso de profissionais não médicos, o uso terapêutico de $\mathrm{PM}^{17}$. Em contrapartida, outros consideraram essa forma de origem do conhecimento superficial, optando por não prescreverem sem haver aprofundamento científico na temática ${ }^{21}$.

As pesquisas demonstram que a limitação de entendimento ocorre por uma falha estrutural dos planos de ensino acadêmico e das instituições por não oferecerem cursos de capacitação com o tema ou incentivarem os profissionais a buscar esses conhecimentos ${ }^{21}$. Contudo, como demonstram Alonso-Castro e colaboradores ${ }^{20}, 73 \%$ da amostra de profissionais possuíam interesses em adquirir informações, via cursos de capacitação, materiais complementares ou outras fontes que abordassem a utilização de $\mathrm{PM}$ na $\mathrm{APS}^{17,21}$.

Outro aspecto importante abordado pelos artigos estudados é a importância de saber qual a visão dos profissionais em relação ao uso de PM, uma vez que a opinião pessoal reflete na prática profissional.

Alonso-Castro et al. apontam que $46 \%$ dos profissionais de saúde e $51 \%$ dos médicos entrevistados acreditavam que os pacientes não deveriam fazer uso de PM por motivos como ineficácia, falta de embasamento científico e falta de segurança. Porém, $54 \%$ desses profissionais de saúde e $49 \%$ dos médicos já fizeram uso pessoal de PM como terapia alternativa, alegando eficácia, tradição familiar e destacando a redução do consumo de medicamentos alopáticos ${ }^{20}$. Ou seja, os profissionais de saúde têm receio de prescrever, mas acabam usando para tratamento próprio.

Dos profissionais entrevistados no RS, $81,4 \%$ reconheceram que a inclusão de políticas que visam à inserção do uso de PM na APS seria de suma importância ${ }^{18}$. Contudo, alguns acreditam que a utilização das PM é eficaz apenas como forma de autocuidado em doenças em estágio inicial e de forma adjuvante em doenças paliativas ${ }^{21}$.

Quanto ao autocuidado, é importante salientar que o potencial de desmedicalização apresentado pelas PICs não independe da maneira como são aplicadas e utilizadas: a experimentação e abordagem propostas devem estimular uma ampliação da liberdade do paciente para sentir, refletir, experimentar e se conhecer para alcançar um modelo de autocuidado ${ }^{22}$. Por isso, Macià e colaboradores acrescentam que não se pode esquecer do caráter essencialmente popular desse conhecimento, mostrando abertura para aprender e construí-lo junto à população ${ }^{23}$.

Nesse sentido, Souza et al. sugerem que a inserção das PM na APS se tornaria efetiva se o profissional de saúde organizasse grupos de discussão com os usuários da USF, exercendo diálogo interdisciplinar, praticando educação em saúde e entendendo o contexto no qual encontra-se inserido $^{18}$. Dessa forma, o agir profissional se estabelece embasado em uma relação de respeito às crenças e aos valores do indivíduo, empoderado 
no seu saber histórico e popular, na forma de um diálogo em que ambas as partes aprendem acerca da cidadania e da heterogeneidade do cuidado, fugindo da lógica do atender apenas a doença $\mathrm{a}^{24,25}$.

\section{Usuários de PM na APS: perfil e fatores que levam ao uso}

Colet e colaboradores, por meio de entrevistas aplicadas a 446 usuários de unidades de saúde da cidade de Ijuí (RS), mostraram que $81 \%$ dos entrevistados utilizam PM e 71,5\% afirmam utilizá-las por indicações de parentes, 44,6\% deles se encontram na faixa etária de 40 a 59 anos e 67,7\% com renda de até um salário-mínimo e meio ${ }^{26}$.

Outro estudo, de Zeni et al., entrevistou 701 indivíduos de Blumenau (SC) e observou que a maioria dos que utilizavam PM eram mulheres $(78,1 \%)$, brancas $(81,6 \%)$, casadas $(56,4 \%)$ e pertencentes à classe $\mathrm{C}(56,4 \%)$, sendo a média de idade de 43,3 $\operatorname{anos}^{27}$.

Em 2016, Szerwieski e colaboradores entrevistaram 252 idosos em Itaipulândia (PR), também mostrando o uso mais prevalente em mulheres $(67,58 \%)$, com pouca (até quatro anos de estudo) ou nenhuma escolarização (90,11\% dos que usam), não sendo economicamente ativas $(83,52 \%)$ e recebendo até um salário-mínimo mensal $(97,25 \%)^{28}$.

Os dados de Colet et al., Szerwieski et al. e Zeni et al. evidenciam o legado de uma sociedade patriarcal, em que a mulher se tornou a responsável pela manutenção doméstica e pelo cuidado dos familiares ${ }^{29}$, bem como pelo cultivo de plan$\operatorname{tas}^{30}$. Com isso, as mulheres normalmente têm mais informações sobre as PM e acabam recorrendo a elas, por poderem ser obtidas de maneira econômica e acessível em seus próprios quintais ${ }^{31}$.

Estudo de Dantas e colaboradores entrevista profissionais da saúde e representantes do MST, fazendo uso de grupos focais com famílias e diário de campo, ressalta o papel de se incentivar o uso de PM e fortalecer o saber popular nos dois assentamentos pesquisados por meio de núcleos locais $^{32}$. Esse cuidado vai ao encontro de experiências do MST relatadas por setores estaduais da saúde, no sentido de promoção de hábitos saudáveis, efetivação do direito à saúde e preparo de remédios à base de $\mathrm{PM}^{33}$.

Em outra realidade, $\mathrm{o}$ artigo de Macià et al., realizado em Barcelona, Espanha, entrevistou 161 indivíduos, encontrando que $85 \%$ usavam PM, sendo 65,7\% mulheres, e 45,2\% eram analfabetos ou cursaram apenas o primeiro grau. Embora a idade média da amostra tenha sido de
62,9 anos, a faixa etária de 15-40 anos (14\% do total) foi a que mais referiu usar PM (97\%), enquanto no grupo de 65 anos ou mais esse valor foi de $48,2 \%$. Com isso, os resultados em números absolutos apontam que o perfil de usuário de PM é de pessoas acima dos 65 anos, analfabetos ou que cursaram apenas o ensino fundamental ${ }^{23}$.

No Peru, levando em conta a diversidade cultural e étnica do país, Valenzuela-Oré e colaboradores $^{34}$ abordam a temática social do uso de PM. Aplicaram-se questionários a 775 povoados urbanos e rurais, sendo que $68,7 \%$ dos entrevistados relataram sempre usar $\mathrm{PM}^{34}$. No entanto, nota-se diminuição no uso da medicina tradicional na população peruana ${ }^{35}$.

Já em estudo no Paquistão, Yaseen et al. mostraram que a população local entrevistada fazia uso de PM pela falta de serviços de saúde adequados para o tratamento de diversas comorbidades, além de ser uma herança cultural e tradicional indígena ${ }^{36-38}$.

Outro artigo encontrado difere dos anteriores e relata a construção do documentário "Cantos e saberes", partindo da pesquisa "Quintais produtivos e sua relação com a segurança alimentar e nutricional"39. Para a construção do documentário, os autores realizaram uma pesquisa com o intuito de analisar saberes e práticas tradicionais do uso de PM entre as mulheres frequentadoras do Grupo Horta, em Piraquara (PR).

O documentário é uma modalidade que traz a possibilidade de diálogo entre esferas distintas - com profissionais de saúde, pesquisadores e comunidade - e suas realidades ${ }^{40,41}$, apontando para o potencial humanizador de práticas integrativas e complementares (PIC), como as PM, no cuidado em saúde, ao mesmo tempo em que ressalta as repressões políticas e mercantis que elas sofrem ${ }^{42}$.

Por sua vez, Tribess et al., na região de mata atlântica do estado de Santa Catarina, fizeram um levantamento etnobotânico com os moradores locais. Notou-se que a prática da medicina tradicional em comunidades rurais é uma fonte importante de autocuidado, diretamente ligada aos princípios da APS no manejo de patologias recorrentes.

Esse estudo cita que a mata atlântica possui uma das maiores biodiversidades de PM por metro quadrado, por isso favorece o acesso a diversas plantas. Isso poderia servir de ferramenta estratégica para práticas de educação em saúde e de estímulo à preservação florestal, propiciando um fortalecimento significativo das políticas nacionais de uso de PM e beneficiando diretamente a população usuária do SUS ${ }^{43}$. 
Por fim, a análise de Caccia-Bava e colaboradores, feita em 4.249 unidades de saúde do estado de São Paulo, por meio dos dados do Primeiro Ciclo do Programa Nacional de Melhoria do Acesso e da Qualidade da Atenção Básica (PMAQ), evidenciou o uso de PM mais prevalente em municípios de grande porte e com indicadores socioeconômicos mais altos (63,9\% dos classificados nos estratos 4, 5 e 6 do PMAQ), seguidos pelas cidades menores e marginalizadas (55,7\% das classificados nos estratos 1 , 2 e 3 do PMAQ) ${ }^{44}$.

Assim, explicita-se a necessidade de expandir esse conhecimento de forma mais abrangente na comunidade e na equipe de saúde, no intuito de contemplar a população de maneira homogênea, para que usufruam dos recursos naturais e sustentáveis que seus territórios estão aptos a lhes fornecer, sempre preservando e respeitando os aspectos culturais da localidade ${ }^{12}$.

\section{A falta de segurança no uso de PM}

Alguns estudos apontam as dificuldades e os problemas associados à falta de conhecimento dos usuários sobre a segurança ou os riscos no uso de PM.

No trabalho de Szerwieski et al., mostrou-se que usuários não sabiam corretamente as contraindicações e os possíveis efeitos colaterais, e disseram fazer uso indiscriminado, acreditando que por ser algo mais natural não poderia apresentar malefícios ${ }^{28}$. Soria e colaboradores relatam que no Paraguai a identificação correta das espécies é complicada porque a comercialização das mesmas se dá pelo nome popular, podendo gerar confusões ${ }^{45}$. Eles então discutem que a inclusão de PM na APS deve ser pautada pelo uso seguro e eficaz, com a correta identificação da espécie, de sua origem, seu preparo e a dose adequada para cada caso ${ }^{45}$. Por isso, é essencial identificar, por meio de estudos farmacológicos e toxicológicos, os possíveis riscos acerca dessa prática, bem como fornecer informações cientificamente embasadas aos profissionais de saúde, estabelecendo um programa de farmacovigilância ${ }^{20}$.

Além disso, o estudo Caccia-Bava et al. revelou que no estado de São Paulo apenas 5,1\% do total de 467 unidades de saúde que disponibilizam fitoterápicos o fazem com a planta in natura ${ }^{44}$.

No Brasil, uma possibilidade de ampliação institucionalizada e segura do uso de PM na APS é o programa Farmácia Viva, um programa de assistência social farmacêutica que prepara, prescreve e dispensa fitoterápicos na rede pública de saúde. Também orienta sobre o uso de plantas, garantindo eficácia e segurança a partir de jardins medicinais com certificação botânica ${ }^{21}$.

Com a implementação da Farmácias Viva na APS, é possível trabalhar com conhecimentos e usos populares das plantas, associando-os a evidências científicas, bem como produzir remédios fitoterápicos seguros para uso da população, considerando também efeitos colaterais e contraindicações de cada um deles.

Em relação a quais PM mais são utilizadas, os trabalhos mostraram uma variabilidade muito grande, a depender de localidade geográfica, cultura e fatores históricos e econômicos ${ }^{20,26,36,45-47}$.

Como limitação da presente revisão integrativa, foi evidenciada a escassez de estudos que descrevam o conhecimento da população em geral e dos profissionais de saúde sobre eficácia, formas de administração, interações medicamentosas e efeitos adversos das PM utilizadas no Brasil e no mundo.

\section{Considerações finais}

As evidências científicas relacionadas às formas de uso de plantas medicinais na atenção primária à saúde caracterizam os usuários e trazem dados acerca do conhecimento dos profissionais e da segurança dessas. Conclui-se que, na atenção primária à saúde, o perfil de usuário de plantas medicinais é constituído por mulheres, idosas, com baixas escolaridade e renda, tanto no Brasil quanto em outros países, o que evidencia o papel das mulheres na produção, no uso e na transmissão de conhecimentos tradicionais.

Também se evidencia que, durante a formação dos profissionais da saúde, os temas de práticas integrativas e complementares não são abordados, gerando menos conhecimento, mais preconceito por falta de informação e levando a menos pesquisas, o que resulta em menor incentivo e divulgação para a população.

Nesse contexto, é difícil promover o uso seguro, cientificamente embasado e eficaz das PM na APS, uma vez que se tem como limitantes o desconhecimento acerca de como se identificar corretamente as plantas, da forma adequada de uso, da dose recomendada e das possíveis interações medicamentosas, efeitos adversos e toxicidades.

Porém, com formação profissional adequada, a APS tem potencial para desenvolver hortas medicinais ou implantar Farmácias Vivas, garantindo acesso às PM de forma segura e eficaz e atuando junto dos saberes populares na melhoria do bem estar e do autocuidado da população. 


\section{Colaboradores}

KP Patrício e GC Barbosa participaram do delineamento do projeto, da análise dos resultados, da escrita e revisão do manuscrito. ACS Minato, MA Lopes, AF Brolio e GR Barros contribuíram em todas as fases da pesquisa, incluindo levantamento de dados e literatura, tabulação, análise dos resultados, escrita e revisão do manuscrito. VS Moraes trabalhou no levantamento de dados e literatura, na formatação e revisão final das referências.

\section{Referências}

1. Firmo WCA, Menezes VJM, Passos CEC, Dias CN, Alves LPL, Dias ICL, Santos Neto M, Olea RSG. Contexto histórico, uso popular e concepção científica sobre plantas medicinais. Cad Pesq São Luís. 2011; 18(especial):90-95.

2. Oliveira LST, Cunha e Silva SL, Tavares DC, Santos A, Oliveira GCB. Uso de plantas medicinais no tratamento de animais. Encicl Biosfera 2009; 5(8):1-8.

3. Brasil. Ministério da Saúde (MS). Secretaria de Ciência, Tecnologia e Insumos Estratégicos. Departamento de Assistência Farmacêutica e Insumos Estratégicos. Uso de medicamentos e medicalização da vida: recomendações e estratégias. Brasília: MS; 2019.

4. Agência Nacional de Vigilância Sanitária (Anvisa). Medicamentos fitoterápicos e plantas medicinais [Internet]. Brasília: Anvisa. [acessado 2020 Ago 5]. Disponível em: http://portal.anvisa.gov.br/fitoterapicos

5. Koifman L. O modelo biomédico e a reformulação do currículo médico da Universidade Federal Fluminense. Hist Cienc Saúde Manguinhos 2001; 8(1):49-69.

6. Caponi S, Verdi M, Brzozowski FS, Hellmann F, organizadores. Medicalização da Vida: Ética, Saúde Pública e Indústria Farmacêutica. Cien Saude Colet 2012; 17(9):2553-2556.

7. Harayama R, Gomes J, Barros R, Galindo D, Santos D. Fórum sobre Medicalização da Educação e da Sociedade. Nota técnica: o consumo de psicofármacos no Brasil [Internet]. 2015. [acessado 2020 Ago 5]. Disponível em: http://medicalizacao.org.br/nota-tecnica/

8. Ibiapina WV, Leitão BP, Batista MM, Pinto DS. Inserção da fitoterapia na atenção primária aos usuários do SUS Rev Ciênc Saúde Nova Esperança 2014; 12(1):58-68.

9. Relatório final da $8^{a}$ Conferência Nacional de Saúde [Internet]. Brasília; 1986 Mar 17-21. [acessado 2020 Ago 5]. Disponível em: https://bvsms.saude.gov.br/ bvs/publicacoes/8_conferencia_nacional_saude_relatorio_final.pdf

10. Brasil. Ministério da Saúde (MS). Secretaria de Atenção à Saúde. Departamento de Atenção Básica. Politica Nacional de Práticas Integrativas e Complementares no SUS - PNPIC-SUS. Brasília: MS; 2006.
11. Brasil. Ministério da Saúde (MS). Secretaria de Ciência, Tecnologia e Insumos Estratégicos. Departamento de Assistência Farmacêutica. Política nacional de plantas medicinais e fitoterápicos. Brasília: MS; 2006.

12. Figueiredo CA, Gurgel IGD, Gurgel GD Jr. A Política Nacional de Plantas Medicinais e Fitoterápicos: construção, perspectivas e desafios. Physis 2014; 24(2):381-400.

13. Antonio GD, Tesser CD, Moretti-Pires RO. Contribuições das plantas medicinais para o cuidado e a promoção da saúde na atenção primária. Interface (Botucatu) 2013; 17(46):615-633.

14. Ganong LH. Integrative reviews of nursing research Res Nurs Health 1987;10(1):1-11.

15. Whittemore R, Knafl K. The integrative review: updated methodology. J Adv Nurs 2005; 52(5):546-53.

16. Minayo MCS. $O$ desafio do conhecimento: pesquisa qualitativa em saúde. São Paulo: Hucitec; 2013

17. Mattos G, Camargo A, Souza CA, Zeni ALB. Plantas medicinais e fitoterápicos na Atenção Primária em Saúde: percepção dos profissionais. Cienc Saude Colet 2018; 23(11):3735-3744.

18. Souza ADZ, Heinen HM, Amestoy SC, Mendieta MC, Piriz MA, Heck RM. O Processo de trabalho dos enfermeiros da atenção primária e a Política de Plantas Medicinais/Fitoterápicos. Rev Bras Plantas Med 2016; 18(2):480-487.

19. Soares DP, Coelho AM, Silva LEA, Silva RJR, Figueiredo CR, Fernandes MC. Política nacional de práticas integrativas e complementares em saúde: discurso dos enfermeiros da atenção básica. Rev Enferm Centro-Oeste Min 2019; 9:e3265.

20. Alonso-Castro AJ, Domínguez F, Maldonado-Miranda JJ, Castillo-Pérez LJ, Carranza-Álvarez C, Solano E, Isiordia-Espinoza MA, Del Carmen Juárez-Vázquez M, Zapata-Morales JR, Argueta-Fuertes MA, Ruiz-Padilla AJ, Solorio-Alvarado CR, Rangel-Velázquez JE, Ortiz-Andrade R, González-Sánchez I, Cruz-Jiménez G, Orozco-Castellanos LM. Use of medicinal plants by health professionals in Mexico. J Ethnopharmacol 2017; 198:81-86. 
21. Palma JS, Badke MR, Heisler EV, Hick RM, Meincke SMK. Modelos explicativos do setor profissional em relação às plantas medicinais. Rev Pesqui Cuid Fundam 2015; 7(3):2998-3008.

22. Tesser Charles Dalcanale, Dallegrave Daniela. Complementary and alternative medicine and social medicalization: lack of definitions, risks, and potentials in primary healthcare. Cad Saude Publica 2020; 36(9):e00231519.

23. Maciá MCD, Grau PE, Carrobé EF. Consumo de plantas medicinales em um barrio de Barcelona: lanecesidad de registrar informáticamente este hábito. Rev Fitoter 2016; 16(1):57-64.

24. Boehs AE, Monticelli M, Wosny AM, Heidemann IBS, Grisotti M. A interface necessária entre enfermagem, educação em saúde e o conceito de cultura. Texto Contexto Enferm 2007; 16(2):307-314.

25. Langdon EJ, Wiik FB. Antropologia, saúde e doença: uma introdução ao conceito de cultura aplicado às ciências da saúde. Rev Lat Am Enfermagem 2010; 18(3):459-466

26. Colet CF, Cavalheiro CAN, Dal Molin GT, Cavinatto AW, Schiavo M, Schwambach KH, Oliveira KR. Uso de plantas medicinais por usuários do serviço público de saúde do município de Ijuí/RS. Rev Bras Med Fam Comun 2015; 10(36):1-13.

27. Zeni ALB, Parisotto AV, Mattos G, Helena ETS. Utilização de plantas medicinais como remédio caseiro na Atenção Primária em Blumenau, Santa Catarina, Brasil. Cienc Saude Colet 2017; 22(8):2703-2712.

28. Szerwieski LLD, Cortez DAG, Bennemann RM, Silva ES, Cortez LER. Uso de plantas medicinais por idosos da atenção primária. Rev Eletron Enferm 2017; 19:111 .

29. Almeida AV, Tavares Mafra SC, Silva EP, Kanso S. A feminização da velhice: em foco as características socioeconômicas, pessoais e familiares das idosas e o risco social. Texto Contexto Enferm 2015; 14(1):115-131.

30. Vasconcelos MKP, Lima ARA, Barbieri RL, Heck RM. Medicinal plants used by octogenarians and nonagenarians from a small village in Rio Grande/RS, Brazil. Rev Enferm UFPE 2011; 5(6):1329-1336.

31. Oliveira ER, Menini Neto L. Levantamento etnobotânico de plantas medicinais utilizadas pelos moradores do povoado de Manejo, Lima Duarte - MG. Rev Bras Plantas Med 2012; 14(2):311-320.

32. Dantas ACMTV, Martelli PJL, Albuquerque PC, Sá RMPF. Relatos e reflexões sobre a atenção primária à saúde em assentamentos da reforma agrária. Physis 2019; 29(2):e290211.

33. Movimento dos Trabalhadores Rurais Sem Terra (MST). Secretaria Nacional. Relatos de experiência em saúde. Brasília: MST; 2005.

34. Valenzuela-Ore F, Romaní-Romaní F, Monteza-Facho BM, Fuentes-Delgado D, Vilchez-Buitron E, Salaverry-García O. Prácticas culturales vinculadas al cuidado de la salud y percepción sobre la atención em establecimientos de salud en residentes de centros poblados alto-andinos de Huancavelica, Perú. Rev Peru Med Exp Salud Publica 2018; 35(1):84-92.

35. De la Cruz MG, Malpartida SB, Santiago HB, Jullian $\mathrm{V}$, Bourdy G. Hot and cold: medicinal plant uses in Quechua speaking communities in the high Andes (Callejón de Huaylas, Ancash, Perú). J Ethnopharmacol 2014; 155(2):1093-1117.
36. Yaseen G, Ahmad M, Sultana S, Alharrasi AS, Hussain J, Zafar M, Shafiq-Ur-Rehman. Ethnobotany of medicinal plants in the Thar Desert (Sindh) of Pakistan. J Ethnopharmacol 2015; 163:43-59.

37. Lentini F, Di Martino A, Amenta R. Le piante di uso popolar em ell arcipelago dele Pelagie (Ag). L uomo L ambient 1995; 19:117-121.

38. Sanz-Biset J, Campos-de-la-Cruz J, Epiquién-Rivera MA, Cañigueral S. A first survey on the medicinal plants of the Chazuta valley (Peruvian Amazon). J Ethnopharmacol 2009; 122(2):333-362.

39. Starosta JA, Anjos MCR. "Cantos e saberes": processo de construção de um documentário sobre plantas medicinais. RECIIS 2020; 14(1):199-211.

40. Costa MHBV. Cinema e construção cultural do espaço geográfico. Rev Bras Estud Cin Audiov 2013; 2(3):251-262.

41. Black K, Lipscomb VB. The promise of documentary theatre to counter ageism in age-friendly communities. J Aging Stud 2017; 42:32-37.

42. Azevedo E, Pelicioni MCF. Práticas integrativas e complementares de desafios para a educação. Trab Educ Saúde 2011; 9(3):361-378.

43. Tribess B, Pintarelli GM, Bini LA, Camargo A, Funez LA, de Gasper AL, Zeni AL. Ethnobotanical study of plants used for therapeutic purposes in the Atlantic Forest region, Southern Brazil. J Ethnopharmacol 2015; 164:136-146.

44. Caccia-Bava MCGG, Bertoni BW, Pereira MAS, Martinez EZ. Disponibilidade de medicamentos fitoterápicos e plantas medicinais nas unidades de atenção básica do Estado de São Paulo: resultados do Programa Nacional de Melhoria do Acesso e da Qualidade da Atenção Básica (PMAQ). Cienc Saude Colet 2017; 22(5):1651-1651.

45. Soria N, Ramos P. Uso de plantas medicinales em la atención primaria de Salud em Paraguay: algunas consideraciones para su uso seguro y eficaz. Mem Inst Investig Cienc Salud 2015; 13(2):8-17.

46. Picking D, Delgoda R, Younger N, Germosén-Robineau L, Boulogne I, Mitchell S. TRAMIL ethnomedicinal survey in Jamaica. J Ethnopharmacol 2015; 169:314-327.

47. Zivkovic J, Ilic M, Savikin K, Zdunic G, Ilic A, Stojkovic $D$. Traditional use of medicinal plants in South-Eastern Serbia: ethnopharmacological investigation on the current status and comparison with half a century old data. Front Pharmacol 2020; 11:1020.

Artigo apresentado em 11/09/2020

Aprovado em 09/02/2021

Versão final apresentada em 11/02/2021

Editores-chefes: Romeu Gomes, Antônio Augusto Moura da Silva 